\title{
Sexuality and Life-cycle of the Edible, Wild Agaricus bitorquis
}

\author{
By CARLENE A. RAPER \\ Harvard University Biological Laboratories, \\ Cambridge, Massachusetts 02138, U.S.A.
}

(Received 2I August 1975; revised 17 November 1975)

SUMMAR Y

Five collections from nature, originally identified as representing the three species Agaricus campestris, A. edulis and A. bitorquis, have been shown to be interfertile and are therefore designated $A$. bitorquis (Quélet) Saccardo. All stocks can be fruited, and analysis of the life-cycle has revealed heterothallism with sexuality controlled by a single incompatibility locus with multiple alleles: monosporous siblings are self-sterile and cross-fertile in a bipolar pattern. The fertile interaction in compatible matings is morphologically distinguishable in the formation of a differentiated dikaryotic mycelium without the clamp connexions common in the dikaryons of other Hymenomycetes. The segregation pattern of incompatibility alleles and nutritional markers indicates haploidy of vegetative nuclei. Six parental and four rare, non-parental incompatibility types were identified in the sample studied. Heterokaryotic interactions-dikaryons with homokaryons and dikaryons with dikaryons to establish new dikaryons - have been shown to occur. A remote kinship to the cultivated mushroom was demonstrated in the formation of transitory heterokaryons between $\mathrm{A}$. bitorquis and $\mathrm{A}$. bisporus, but these could not be fruited. The life-cycles and patterns of sexuality in the two species are compared and the possibility that $A$. bitorquis could be an alternative to $A$. bisporus for mass cultivation and consumption, with emphasis on the advantage in breeding $A$. bitorquis, is mentioned.

\section{INTRODUCTION}

The functional relationships among members of the genus Agaricus have not been explored. The origin of the commonly cultivated bisporic mushroom remains a mystery, and its relationship to the predominating tetrasporic forms found in nature is unknown. A knowledge of life-cycles and interfertility patterns within the genus is requisite to an understanding of species and their possible kinships.

The life-cycle and sexual nature of the cultivated Agaricus bisporus have recently been described (Miller, I97I; Raper, Raper \& Miller, 1972; Miller \& Kananen, 1972; Raper \& Raper, 1972; Elliott, 1972). The first comprehensive biological characterization of a wild, tetrasporic species of Agaricus is reported here. Life-cycles and sexual interactions were analysed in five collections, and the relationship between these collections and $A$. bisporus was examined. Although the five collections were originally designated as representing three species, all have been found to comprise a single species, presently designated Agaricus bitorquis (Quélet) Saccardo. The species is edible, tasty, cultivatable and of possible interest to commercial mushroom growers.

\section{METHODS}

Stocks. The five collections of Agaricus from nature that were examined in this study are listed here as stocks I to 5 , with the species identification as originally designated. 
Stock I: Agaricus edulis, mycelium derived from tissue culture of a sporophore (sporophore culture), collected in Illinois, U.S.A. in 1959, and identified by C. Bretzloff, Campbell Institute for Agricultural Research, Riverton, New Jersey, U.S.A.

Stock 2: A. bitorquis, sporophore culture, collected in Horst, The Netherlands in 1968 by H. Visscher and M. Vaandrager, identified by C. Bas, Rijksherbarium, Leiden, The Netherlands, and supplied by G. Fritsche, Stichting Proefstation voor de Champignon Cultuur, Horst.

Stock 3: A. bitorquis, sporophore culture, collected in Amsterdam, The Netherlands in I 968 by M. Hagen-Berghuys, identified by C. Bas, and supplied by G. Fritsche.

Stock 4: A. campestris, spore print, collected in St Charles, Illinois in 1972, and identified by W. Robbins, Campbell Institute for Agricultural Research.

Stock 5: Psalliota edulis (= Agaricus edulis), sporophore culture, collected in Ghent, Belgium in 1964 by J. A. Poppe, Laboratoria voor Fytopathologie en voor Fytovirologie, Rijksuniversiteit-Gent, and identified by the collector and P. Heinemann, Agricultural University of Gembloux, Belgium (Poppe, 1972).

One representative dikaryon of a cross between progeny of stocks I and 4, with incompatibility types $A I$ and $A 4$, has been deposited in the American Type Culture Collection, listed as ATCC24666, Agaricus edulis.

Cultural techniques. The standard complete and minimal media used for the analysis of A. bisporus (Raper et al., 1972) were employed for vegetative culturing throughout this study. Cultures were usually incubated at the optimal temperature of $30^{\circ} \mathrm{C}$ and stored at 4 to $5{ }^{\circ} \mathrm{C}$.

Analysis of the five collections of Agaricus was begun by culturing single-spore isolates of each stock. When not immediately available, the spores were obtained by fruiting the mycelium derived from sporophore tissue. Spores were collected on sterile paper and, after suspension in sterilized distilled water, were spread on complete medium. Germination was achieved by incubating the spore spread for 5 to $2 \mathrm{I}$ days at $24{ }^{\circ} \mathrm{C}$ in the environment of a mature mycelium of Agaricus. The spore spread was inverted over a plate containing week-old culture from seven evenly distributed inocula of the $W$ strain of $A$. bisporus (Raper et al., 1972). The occasional contaminants of a bacterial and Ascomycetous nature were eliminated by incorporating $50 \mu \mathrm{g}$ penicillin $/ \mathrm{ml}, \mathrm{I} 5 \mu \mathrm{g}$ streptomycin $/ \mathrm{ml}$ and $5 \mu \mathrm{g}$ benomyl/ $\mathrm{ml}$ in the medium. Single germlings and hyphal-tip cells were isolated with a cutting device mounted on the objective of a compound microscope (Raper, 1963), and were transferred to fresh complete medium for isolated growth.

Mating techniques. Matings were made as previously described for A. bisporus (Raper et al., 1972) and were routinely incubated for 2 weeks at $30^{\circ} \mathrm{C}$ plus 2 weeks at $24{ }^{\circ} \mathrm{C}$. The cultures were observed for evidence of interaction two or three times over this period of incubation. In the tests for identification of incompatibility type, the observation of a positive, compatible interaction (see the following section) was interpreted as indicating a difference in the incompatibility types of the two mated individuals. A lack of interaction was interpreted more cautiously: the mating test was repeated with several testers known to be of an identical incompatibility type, and the tested individual was assigned to that type only after it repeatedly failed to interact with the several testers. No assignment of incompatibility type was made when a tested individual failed to interact with all testers of more than one incompatibility type.

Observation of nuclei. The nuclear content of cells was determined by phase microscopic observation of mycelia grown for I week on sterilized permeable cellophane membrane (Dupont no. I50 PD 62) laid over solid culture medium in a Petri dish. For observation, 

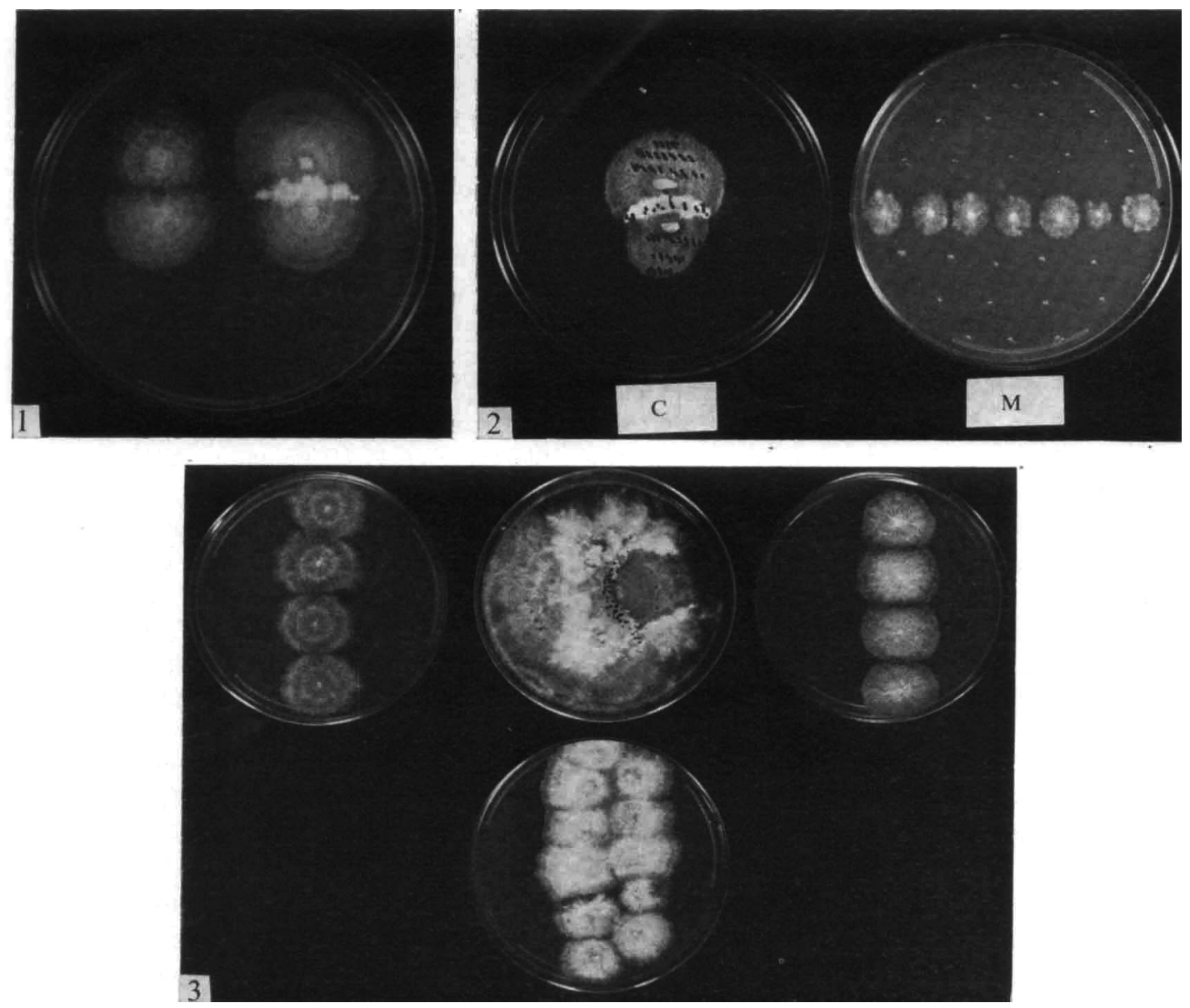

Fig. I. A negative versus a positive mating interaction in $A$. bitorquis is illustrated by the pairing of siblings in an incompatible combination (left) and a compatible combination (right). A visibly distinct mycelium is formed in the zone of contact between the two compatible strains.

Fig. 2. Mating between nutritionally complementing auxotrophic homokaryons of $A$. bitorquis. Mycelial plugs were taken in a grid pattern as shown from a 2-week-old mating of $A 2 \mathrm{met}_{\text {-thr }}{ }^{-} \mathrm{his}^{+} \times$ AI met-thr ${ }^{+}$his on complete medium (C) and transferred to corresponding positions on minimal medium (M). Inocula from the morphologically distinct mycelium in the contact zone were the only ones that grew prototrophically.

Fig. 3. Patterned subculture, on complete medium, of a compatible mating of $A$. bitorquis, showing persistence of distinctive morphology of the heterokaryon that developed in the contact zone (below) as compared with the morphologies of those portions of the two mated strains that remained homokaryotic (left and right).

a section was cut from the edge of a colony and placed, mycelial side up, in a drop of $14 \%$ gelatin at melting temperature; another drop of molten gelatin was placed between the mycelium and a coverslip. The nuclei were observed under oil immersion at $2000 \times$ magnification.

Isolation of mutant strains. Mutants with nutritional deficiencies, auxotrophs, were generated in spores using X-rays: $100 \mathrm{kV}$ (Norelco MG ${ }_{100}$ ) in the dosage range 150000 to $250000 \mathrm{R}$ to cause mortality of 90 to $99 \%$ of the spores. The identification of auxotrophs in monosporous cultures was done as described by Raper et al. (1972). 
Table I. Mating interactions among progeny of stock I of A. bitorquis

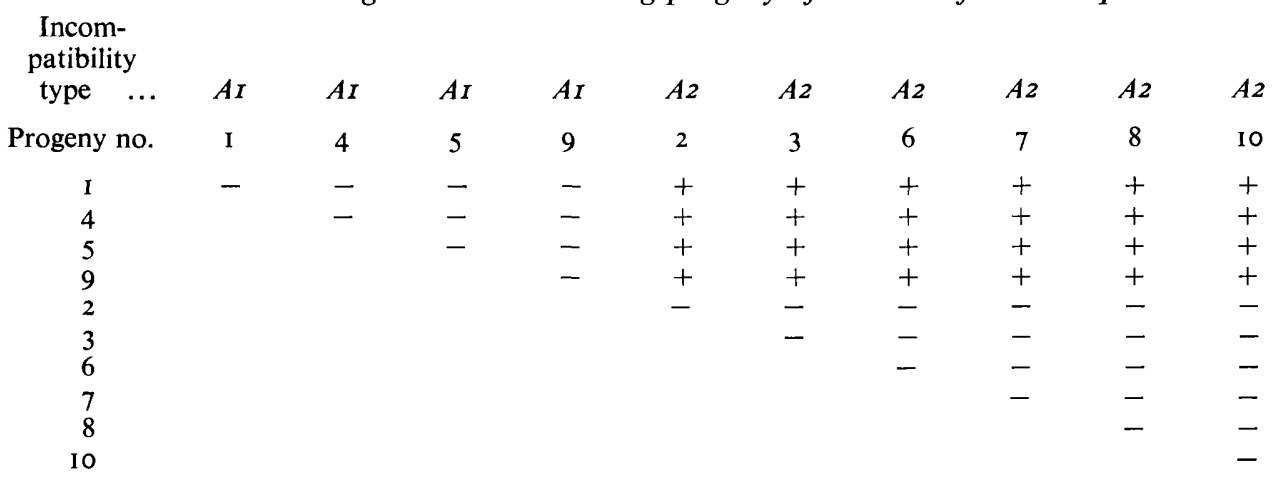

Tests for fruiting. Tests for fertility of mycelia were carried out in the laboratories of the Campbell Institute for Agricultural Research at Riverton, New Jersey, under the supervision of Dr R. E. Miller. Miller employed a modified Till substrate and used the methods described for A. bisporus (Miller \& Kananen, 1972) except that the temperature was elevated by $6{ }^{\circ} \mathrm{C}$ after casing.

\section{RESULTS}

Analysis of one stock

Evidence for a pattern of sexuality in wild Agaricus was sought first by analysis of progeny from a single stock, stock I from Illinois.

Evidence for heterothallism and bipolar incompatibility. Heterothallism was indicated by the development of a morphologically distinct type of mycelium in certain matings between siblings. The distinct mycelium occurred along the line of meeting of the two mated strains and appeared whiter and fluffier than that of the strains in isolation, of self-paired strains, or of mated siblings in which no interaction occurred (Fig. I). The pattern of occurrence of such positive interactions in intersib pairings implicated a single genetic locus with alternate alleles as the basis of control in mating interactions. The locus has conventionally been termed an incompatibility factor, $A$, in other Hymenomycetes, but it will be referred to here as the incompatibility locus $A$. About half of all possible pairings between ten monosporous isolates of stock I produced the distinct mycelium, and no interaction was apparent in the matings with self (Table I). The progeny could be divided into two groups with respect to their mating interactions. Four were of one group and were assigned to the incompatibility type $A r$; the remaining six were of the other group and were assigned to the incompatibility type $A 2$. No pairings within a group interacted to produce the distinct mycelium, i.e. all were incompatible; all pairings between the two groups interacted positively to produce the distinct mycelium and hence were compatible.

Two representatives from each group were chosen as testers in matings with the remaining 20 members of the sample of 30 progeny isolated. Two of the 20 indicated no interaction with any of the testers, but eight were compatible with the two $A 2$ testers only and ten were compatible with the two $A I$ testers only. Of the total of 30 progeny first analysed from stock I, I 2 were assigned to the incompatibility type $A I, \mathrm{I} 6$ to $A 2$, and two could not be assigned to either type. This pattern of segregation for incompatibility types fits the expected pattern for control of sexuality by alternate alleles of a single genetic locus, which is common in other Hymenomycetes and known as unifactorial or bipolar incompatibility. 
Proof of heterokaryosis. The interaction between members of the two incompatibility groups, $A I$ and $A 2$, to produce a morphologically distinct mycelium suggested the interaction of compatible homokaryons to produce heterokaryons. Proof of heterokaryosis required the demonstration that the nuclei of paired strains coexist in the hyphae of a single mycelium developed in the mating. The nuclear labels of choice were nutritionally deficient mutations, recessive to their corresponding wild-type alleles. Three such auxotrophic mutations were recovered from 3500 surviving spores of stock I after a 95 to $99 \%$ lethal dose of $\mathrm{X}$-radiation. All untreated progeny of stock I grew on the minimal medium; the generated auxotrophs required the addition of one of the following nutrients: histidine, nicotinic acid, or methionine + threonine. Mating-type tests revealed the histidineless (his ${ }^{-}$) and nicotinic acidless (nic ${ }^{-}$) mutants as members of the group $A I$ and the methionineless-threonineless mutant as belonging to the group $A 2$. The double requirement of the methioninelessthreonineless mutant could not be separated into single requirements for either amino acid through crosses with wild-type strains. The mutant may carry a single mutation that blocks the production of homoserine, a precursor of both methionine and threonine (Emerson, I 949). It is therefore symbolized as met-thr- .

Several matings of the three auxotrophs, in the three possible nutritionally complementing pairs, were analysed for evidence of heterokaryosis. Mycelial plugs taken in a grid pattern from 2- to 3-week-old matings on complete medium were transferred to corresponding positions on minimal medium, and observed for growth after a few days incubation. Only the two

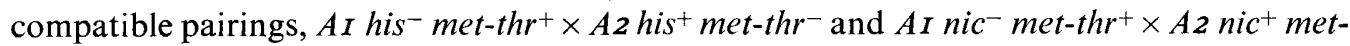
$t h r^{-}$, produced prototrophic mycelia that grew on minimal medium, and in each case the prototrophic mycelium was seen as coincident with the distinct mycelium that developed in the zone of contact of the mated strains (Fig. 2). No prototrophic mycelium appeared in the incompatible mating $\mathrm{AI} h \mathrm{his}^{-} \mathrm{nic}^{+} \times \mathrm{AI} \mathrm{his}^{+} \mathrm{nic}^{-}$, and subsequent attempts to obtain prototrophic mycelia through nutritional selection in incompatible matings of complementing auxotrophic progeny from outcrosses of the three auxotrophs failed in 13 trials.

Prototrophy of the inocula from each of the two compatible matings reflected the association of the two complementing genomes, either in heterokaryosis or in a crossfeeding mixture. A distinction between these two possibilities was sought in analyses of mycelia derived from hyphal-tip isolates of the subcultured prototrophic mycelia. Ninety-nine per cent of the isolated I- to 2-cell hyphal tips in two samples of over I00 each grew as prototophs. It was thus clear that most cells of the distinct, prototrophic mycelium of the compatible matings were heterokaryotic.

Dikaryosis without clamp connexions. The extent of the heterokaryotic mycelium developed in compatible matings of different homokaryons varied from a few spots in the contact zone to a large area over the surface of the mating, and its macroscopically distinct morphology persisted indefinitely in subculture (Fig. 3). It could be distinguished microscopically from homokaryotic, unmated isolates by the nuclear content of cells (Fig. 4). The cells of the heterokaryons examined were predominantly binucleate as compared with the cells of the component homokaryotic strains which had various numbers of nuclei per cell ranging from one to I4, with most cells evenly distributed in the range of two to five nuclei per cell (Fig. 5). This observation, together with the evidence for heterokaryosis in I- to 2-celled hyphal fragments, suggested that the distinct mycelium of compatible matings is a type of structured heterokaryon known as a dikaryon in which each cell contains one nucleus from each strain. Paired nuclei in the cells have been seen to divide conjugately, but no clamp connexions, a feature of dikaryons formed in compatible matings of many other well-defined Hymenomycetes (Boidin, 197I), were seen in this material. 

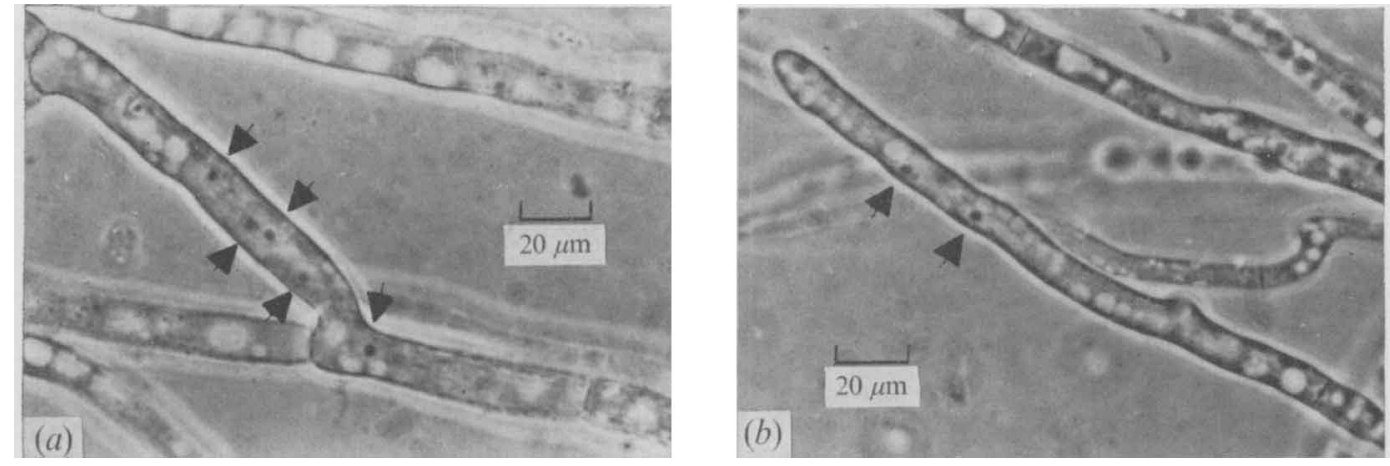

Fig. 4. Comparison of typical homokaryotic and heterokaryotic cells of $A$. bitorquis: a homokaryon with five nuclei $(a)$ and a heterokaryon with two nuclei $(b)$. Arrows point to nuclei. A plug-like structure, typical of the septa, can be seen in the septum at the upper left of the homokaryotic cell $(a)$ and at the lower right of the heterokaryotic cell $(b)$. Photographs were taken under phase-contrast microscopy.

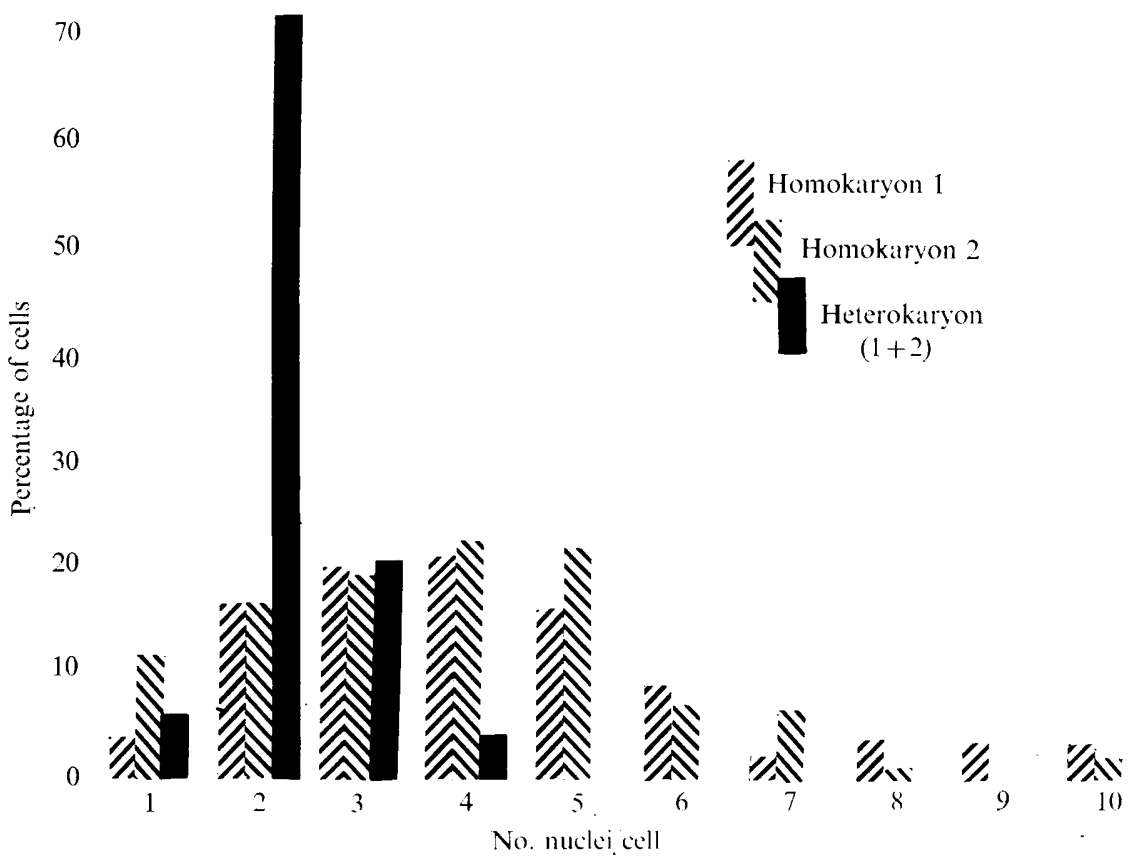

Fig. 5. Nuclear distribution in cells of two homokaryons of stock I (striped bars) versus the heterokaryon (solid bar) derived from the mating of the two homokaryons. The histogram is based on nuclear counts in a sample of Ioo cells of each mycelium. It is representative of data obtained from a total sample of 800 cells from 20 homokaryons and 1000 cells from 40 heterokaryons of stocks I to 5 of A. bitorquis.

Cellular anastomoses between hyphae of all mycelia examined occurred with an incidence of 2 to $3 \%$. Evidence for nuclear migration in compatible matings - another feature common in other Hymenomycetes studied (Raper, 1966)-was not observed. Dikaryons apparently develop from hyphal anastomoses and proliferate by cellular division.

The septa of both homokaryons and dikaryons in this wild species appeared to be 

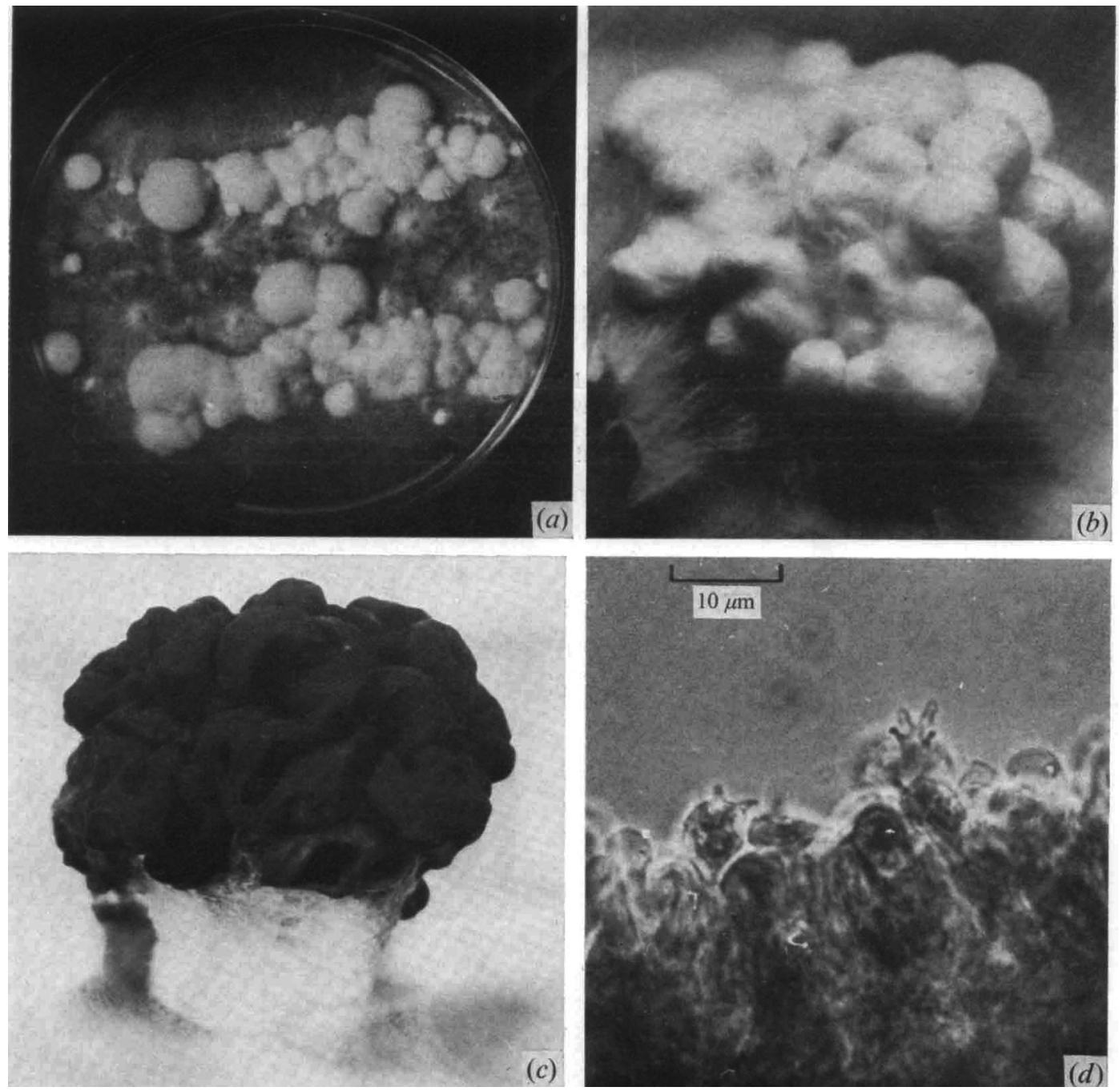

Fig. 6. Amorphous fruiting of a dikaryon of A. bitorquis in Petri dish culture. (a) A culture at two weeks, showing lumps of tightly-packed hyphae; $(b)$ close-up view of a lump that later developed into a fruiting body; $(c)$ an amorphous fruiting body with crinkled, dark-brown spore-bearing surface (hymenium); $(d)$ micrograph of section of hymenium showing the club-like basidia and one basidium with four sterigmata in focus - the spores either have not yet formed or have already been discharged.

similar to those of the mycelia of $A$. bisporus. They had distinct plug-like structures in the middle, and obstructed passage of nuclei. Whether they bear the elaborate structure of the dolipore septum observed by electron microscopy in A. bisporus (Monocha, I965), and in other Hymenomycetes such as Schizophyllum commune (Niederpruem \& Wessels, 1969) and Coprinus lagopus (Geisy \& Day, 1965), is not known.

Dikaryosis and fruiting. Fertility appeared to be correlated with dikaryosis. None of the four unmated progeny of stock I that were subjected to fruiting trials fruited, whereas 17 of the 20 dikaryons tested did. Furthermore, most 2- to 3-week-old dikaryotic cultures on 
Table 2. Segregation ratios of nutritional markers and incompatibility types $A I$ and $A 2$ in intrastock $\mathrm{I}$ crosses of Agaricus bitorquis

\begin{tabular}{|c|c|c|c|c|}
\hline \multirow[b]{2}{*}{ Parental genotypes } & \multirow[b]{2}{*}{ Sample } & \multirow[b]{2}{*}{ Genotypes } & \multicolumn{2}{|c|}{ Segregation $(\%)^{*}$} \\
\hline & & & $A$ I & $A 2$ \\
\hline AI his S $^{-} \mathrm{AL} \mathrm{his}^{+}$ & 176 & $\begin{array}{l}\text { his }^{-} \\
\text {his }^{+}\end{array}$ & $\begin{array}{l}22 \\
2 \mathrm{I}\end{array}$ & $\begin{array}{l}10 \\
47\end{array}$ \\
\hline AI nic ${ }^{+} \times A 2 n i c^{-}$ & 183 & $\begin{array}{l}\text { nic }^{+} \\
n i c^{-}\end{array}$ & $\begin{array}{l}25 \\
21\end{array}$ & $\begin{array}{l}32 \\
22\end{array}$ \\
\hline AI met-thr ${ }^{+} \times A 2$ met-thr ${ }^{-}$ & 59 & $\begin{array}{l}\text { met-thr } r^{+} \\
\text {met-thr }\end{array}$ & $\begin{array}{l}47 \\
14\end{array}$ & $\begin{array}{r}3 I \\
7\end{array}$ \\
\hline AI nic ${ }^{+}$met-thr ${ }^{-} \times A 2$ nic $^{-}$met-thr ${ }^{+}$ & 83 & 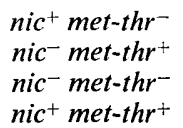 & $\begin{array}{l}16 \\
\text { I I } \\
\text { I } 2 \\
22\end{array}$ & $\begin{array}{r}8 \\
10 \\
6 \\
16\end{array}$ \\
\hline
\end{tabular}

complete medium in Petri dishes formed distinct, raised lumps of tightly packed hyphae which sometimes developed, after an additional 2 weeks of incubation, into amorphous fruiting bodies with four-spored basidia (Fig. 6). Such structures did not form in homokaryotic isolates.

Meiotic segregation and recombination of genetic characters. Analysis of the three nutritionally deficient mutants in fruited crosses of each auxotroph with compatible prototrophic

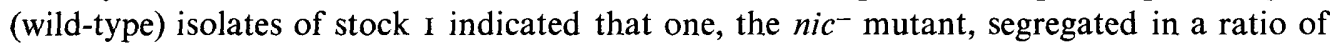
I : I with the wild-type form, nic ${ }^{+}$, among the progeny of the cross (Table 2). This is what would be expected if the mutation was in a single gene and the progeny were haploid and homokaryotic. Segregation of the other two mutants, his ${ }^{-}$and met-thr-, with their respective prototrophic forms, did not fit a I : I ratio: two to four times as many prototrophs as auxotrophs occurred in the offspring of outcrosses of these mutants. A subsequent cross, AI nic $^{+}$met-thr- $\times$A2 nic met-thr $^{+}$, of compatible auxotrophic offspring derived from the earlier crosses, did however yield ratios of segregating nutritional phenotypes that fitted the expected I:I:I:I pattern for the independent segregation of two alleles at each of two loci. The segregation pattern for $A I$ and $A 2$ in these crosses also fitted a I : I ratio very well, and there was no indication for linkage of any of the markers involved (Table 2).

A note of caution on interpretation of these segregation ratios was imposed by the low incidence of spore germination, which ranged from only 0.5 to $5 \%$. This seemed to be characteristic not only of the mutated strains but also of the wild-type strains of this stock; spores from crosses involving only wild-type strains germinated in comparably low incidence. The method of obtaining spores, whether from normal-appearing sporophores developed in the Till substrate or from amorphous spore-bearing bodies on complete medium in Petri dishes, appeared to make no difference to the segregation data obtained.

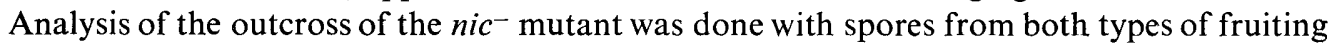
structures and the results were indistinguishable.

The segregation ratios of nutritional markers in two of the crosses, and segregation for the incompatibility markers in all of the crosses, indicated that monosporous progeny of this species are haploid and homokaryotic. The four spores of each basidium appear to constitute a tetrad, with each of the four post-meiotic nuclei contained in a single spore. 
Table 3. Spore germination and incompatibility phenotypes in the interfertile stocks I to 5 of A. bitorquis

\begin{tabular}{|c|c|c|c|c|c|c|c|}
\hline $\begin{array}{c}\text { Stock } \\
\text { no. }\end{array}$ & $\begin{array}{l}\text { Original } \\
\text { identity }\end{array}$ & Collector & Location & $\begin{array}{l}\text { Per- } \\
\text { centage } \\
\text { spore } \\
\text { germi- } \\
\text { nation }\end{array}$ & $\begin{array}{c}\text { Spores } \\
\text { analysed }\end{array}$ & $\begin{array}{l}\text { Incom- } \\
\text { patibility } \\
\text { types }\end{array}$ & $\begin{array}{c}\text { Segregation } \\
\text { ratio } \\
\text { of types } \\
(\%)\end{array}$ \\
\hline I & A. edulis & Bretzloff & Illinois, U.S.A. & $<5$ & $50 \mathrm{I}$ & $A I, A 2$ & $49: 5 \mathrm{I}$ \\
\hline 2 & A. bitorquis & $\begin{array}{l}\text { Visscher \& } \\
\text { Vaandrager }\end{array}$ & Netherlands & 20 & 59 & $A 2, A_{3}$ & $69: 31$ \\
\hline 3 & A. bitorquis & Hagen & Netherlands & 10 & 38 & $A 5, A 6$ & $61: 39$ \\
\hline 4 & A. campestris & Robbins & Illinois & 13 & 64 & $A 2, A 4$ & $34: 66$ \\
\hline 5 & A. edulis & Poppe & Belgium & IO & 97 & $A 2, A_{4}$ & $51: 49$ \\
\hline
\end{tabular}

\section{Confirmation of analysis in other stocks}

Studies similar to that made of stock I of Illinois were done for the four other collections: stocks 2 and 3 of The Netherlands, stock 4 of Illinois, and stock 5 of Belgium. The results were comparable. In each stock, monosporous isolates were self-sterile and cross-fertile in a bipolar pattern: fertile, visibly distinct dikaryons developed regularly in compatible matings, and two incompatibility phenotypes segregated in the samples of 38 to 97 progeny from each of the stocks. Spore germination ranged from to to $20 \%$. The ratio for segregation of incompatibility types was approximately I : I in stocks 3 and 5 but closer to $\mathrm{I}: 2$ in stocks 2 and 4 (Table 3). All the isolates were tested for growth on minimal medium and all proved to be prototrophic.

\section{Relationship between stocks}

Mating interactions and identification of incompatibility phenotypes. Test matings of representatives of each of the two incompatibility types of each stock in all paired interstock combinations revealed interactions between all stocks and multiple allelism at the incompatibility locus. Incompatibility phenotypes in each stock are given in Table 3: stocks 2, 4 and 5 each have one type in common with the $A_{2}$ of stock I; stock 2 has a new type, $A_{3}$; stocks 4 and 5 share not only $A 2$ but a fourth type, $A 4$; both incompatibility types of stock 3 , $A 5$ and $A 6$, are different from those of all the other stocks.

Some progeny of each stock appeared to be sterile or partially sterile in that they did not interact positively with the initial testers of either parental type. When tested with several other representatives of the two types, evidence of compatibility with some members of one type was seen in some cases; others repeatedly failed to interact with testers of either incompatibility type. Tests of the latter group, however, with a large array of representatives of incompatibility types different from those of the parent stock, usually resulted in a few positive interactions. Such 'poor maters', representing 5 to $24 \%$ of the progeny in different stocks, were not assigned incompatibility phenotypes and are not included in the samples given in Table 3.

Non-parental incompatibility types. A low incidence of non-parental $A$ types was detected in the progeny of some crosses. Three of 88 isolates from a cross between nutritionally marked siblings of stock I formed dikaryons in matings with several representatives of both parental $A$ types. They were of two classes; the two members of one class were incompatible with each other but compatible with the one member of the other class. They carried the nutritional markers that went into the cross and were therefore probably not contaminants. One isolate from the sample of 97 progeny of stock 5 was also non-parental in its 
incompatibility type. In addition, one isolate of a cross between descendents of stocks I and 3 , in which a sample of 36 was examined, had a different $A$ type from that of either parent. Although non-parental $A$ types were seen at an incidence of $I$ to 3 per cent in three crosses analysed, none were detected in the total sample of over a thousand progeny of the other two dozen intra- and interstock crosses examined.

All of the non-parental $A$ types proved to be different from the parental $A$ types of all five stocks. With the exception of the two members of the same class from a single cross, the non-parental $A$ types were also different from each other. They were therefore designated $A 7, A 8, A 9$ and $A r o$.

Interstock dikaryons and fertility. The dikaryons that developed in compatible matings between stocks were comparable to those seen in matings within stocks. A sample of 376 cells of 20 different heterokaryons derived from compatible matings between stocks in all possible pairings was compared for nuclear content with a sample of 664 cells of 19 heterokaryons derived from matings within each of the five stocks. The cells were 77 and $70 \%$ binucleate in the interstock and intrastock heterokaryons respectively, and the range in nuclear content of cells for all heterokaryons was I to 4 . Only $26 \%$ of the 818 cells of 20 homokaryons representing all stocks were binucleate, and the range in nuclear content of cells was similar to that illustrated for two homokaryons of stock I in Fig. 4.

The five stocks were completely interfertile. Dikaryons that developed in each of the ten possible interstock matings were subjected to fruiting trials and all produced fruits. Sample size was 2 to 4 in six of the interstock combinations and 16 to 56 in the other four. Trials on the total of 138 interstock dikaryons revealed a higher degree of fertility $-71 \%$ fruited, $25 \%$ pinned (i.e. formed fruiting initials only), $4 \%$ neither fruited nor pinned - than did the trials on IOI intrastock dikaryons in which $53 \%$ fruited, $22 \%$ pinned, and $26 \%$ neither fruited nor pinned.

Only a few interstock crosses, all involving descendents of stocks I and 3, were analysed for segregation and recombination of genetic markers. In each case, the expected parental and recombinant phenotypes for mating type and nutritional character were observed among the progeny. A very high incidence of spore germination ( $>90 \%$ ) was also seen in some of these intrastock crosses.

Reconciliation of nomenclature. On the basis of interfertility, the five collections of wild forms of Agaricus, originally identified as representing the three species A. campestris, $A$. edulis and $A$. bitorquis, are now considered members of a single species. Dr R. Singer of the Field Museum of Natural History, Chicago, Illinois, who kindly examined spores and photographs of the five stocks studied, concluded: 'The spores and photographs as well as the names given suggest that you have in all stocks... one single species which is correctly called Agaricus bitorquis (Quélet) Saccardo.' He states that A. edulis and A. bitorquis are synonymous and feels that $A$. campestris was wrongly identified. This may have been so, but more recent studies in this laboratory have revealed interfertility of $A$. bitorquis with a number of collections from nature assigned to several non-synonymous species.

\section{Mycelial interactions of dikaryons}

The interaction of dikaryons with homokaryons to produce new dikaryons was demonstrated in Agaricus bitorquis. Six dikaryons homozygous for the met-thr- requirement and heterozygous for the incompatibility types $A I$ and $A 2$, were mated with four nic ${ }^{-}$homokaryons, two of $A r$ and two of $A \boldsymbol{2}$. After three weeks' incubation, small plugs of mycelium were transferred from the contact zone to minimal medium. Inocula from all 24 matings grew prototrophically. Furthermore, dikaryons interacted with dikaryons to generate new 
dikaryons. Four nic ${ }^{-}$dikaryons, produced by mating the compatible nic- homokaryons, were mated with the six met-thr dikaryons, and mycelia that developed in the contact zone were prototrophic in 17 of the 24 matings. In both sets of experiments, the emerging prototrophic mycelia were morphologically distinguishable as dikaryotic and remained stable in culture for a period of at least several weeks. The strains used in these experiments were tested for persistence of auxotrophy after the experiments and all had remained stable. Thus, establishment of dikaryons, newly constituted of complementing auxotrophic nuclei from dikaryotic and homokaryotic mates in one case and from two different dikaryotic mates in the other case, was strongly indicated.

\section{Relationship to $A$. bisporus}

Agaricus bitorquis appears to be only remotely related to A. bisporus. Attempts were made to detect heterokaryosis by nutritional selection of prototrophic mycelia in matings between auxotrophic homokaryons of $A$. bitorquis and auxotrophic homokaryons of $A$. bisporus previously developed and described by Raper et al. (1972). No morphological evidence of interaction was seen, and a prototrophic mycelium was produced in only one of nine matings between $A I h^{-} s^{-}, A I n i c^{-}$and $A 2$ met-thr- of stock I of $A$. bitorquis, and $A 2 W 9$ ade ${ }^{-}$, $A I N I u^{-}$(previously designated $A I N I$ unk ${ }^{-}$) and $A I W$ pro- $^{-}$homokaryons of $A$. bisporus. The prototrophic mycelium developed in the contact zone in the mating between $A 2$ met-

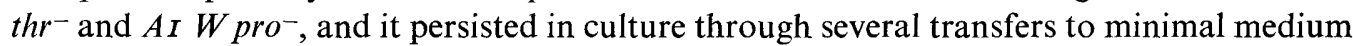
for a period of over a month. (Meanwhile the unmated homokaryons remained stably auxotrophic.) A low incidence of heterokaryosis in cells of the mycelium was revealed in a test for prototrophy of hyphal tips isolated from the mycelium at the end of this period: six of 78 hyphal-tip cultures grew on minimal medium.

Subcultures from the heterokaryotic hyphal fragments did not grow continuously well on minimal medium, however, and could never be made to fruit. One subsequent attempt to mate a his ${ }^{-}$homokaryon of $A$. bitorquis with three different met $^{-}$heterokaryons of $A$. bisporus (strains homozygous for met $^{-}$and heterozygous for mating type, provided by $\mathrm{R}$. E. Miller) produced prototrophic mycelia that were too transient to analyse for heterokaryosis.

Apparently it is possible to establish only transient, infertile heterokaryons between the two species.

\section{DISCUSSION}

Agaricus bitorquis is clearly distinct from the cultivated bisporic species. Its tetrasporic nature has been shown by this study to reflect a significant difference in type of sexuality. Heterothallism, controlled by a single incompatibility locus with multiple alleles, has been revealed by the pattern of self-sterility and cross-fertility of monosporous isolates of the five stocks studied. Segregation ratios of genetic characters have supported previous cytological evidence in tetrasporic forms of Agaricus (Hughes, I96I; Jiři, I965) that karyogamy and meiosis occur in the basidium and that each basidiospore receives a single one of the four post-meiotic nuclei. By contrast, the incompatibility locus of Agaricus bisporus controls a pattern of sexuality known as secondary homothallism, in which self-fertility of monosporous isolates results from the heterokaryotic nature of spores at conception: each of the two basidiospores regularly receives two compatible post-meiotic nuclei carrying different incompatibility alleles (Miller, I97I ; Raper et al., 1972; Miller \& Kananen, 1972; Raper \& Raper, 1972; Elliott, 1972).

The character of the fertile mycelium is another, less significant difference between the species. The fertile mycelium of $A$. bitorquis appears as a morphologically distinct, structured heterokaryon, the dikaryon, in which paired, compatible nuclei are compartmentalized by 
the coupling of cellular division with conjugate nuclear division: a septum is formed perpendicular to the axis of division and separates the paired daughter nuclei. The fertile mycelium of $A$. bisporus, on the other hand, is a heterokaryon with cells containing various numbers of unpaired nuclei, and it cannot be visually distinguished from the homokaryon, which, incidentally, is rarely seen.

The relatedness of the two species is apparent in their common features. Both can be cultivated under comparable conditions, with minor differences, and the mushrooms are generally similar in appearance and taste (Poppe, 1972). Sexuality in the two species is under unifactorial (bipolar) control, the nuclei of the vegetative mycelia are haploid, and the general appearance of monosporous isolates is similar. The optimal temperature for growth and fruiting is a few degrees higher for $A$. bitorquis than for A. bisporus, but this characteristic, along with spore germination, mushroom yield, and detailed morphology of the mushroom, varies considerably within the species and appears to be under genetic control (Raper, 1975).

The inability to establish stable, fertile heterokaryons between $A$. bitorquis and A. bisporus precludes cross-breeding. As for breeding within the species. A. bitorquis has a significant advantage over $A$. bisporus (Raper, 1975). Its heterothallic nature and visibly distinct sexual interaction in compatible matings greatly facilitate the making of controlled crosses to combine characteristics for the development of desirable strains for mass cultivation.

Some features of the basic biology of $A$. bitorquis remain unresolved. The observed sterility of a small percentage of monosporous isolates in cross-matings involving different incompatibility types has not been clarified. It is possibly due to a number of genes similar to those found in Schizophyllum (Raper \& Raper, 1966) that modify heterokaryosis and the sexual process. A genetic analysis of successful outcrosses of partially sterile isolates would be required as a first step in the study of this phenomenon.

The origin of the few non-parental incompatibility types detected in some crosses is open to speculation. Heterokaryosis, diploidy, or disomy for the incompatibility chromosome are unlikely explanations in view of the fact that each non-parental offspring was neither dikaryotic nor self-fertile. Contamination from other stocks present in the laboratory also seems unlikely in view of the fact that the members of the two classes of non-parental $A$ types obtained in the one nutritionally marked cross carried the markers unique to the mated strains of that cross. Spontaneous mutation to a new incompatibility allele would be unprecedented: extensive attempts in related fungi to obtain one incompatibility allele from another by mutation have not been successful. Recombination of two closely linked genes, each with alternate alleles, within the incompatibility locus, however, is a possible explanation that is precedented. Non-parental incompatibility types have been shown to originate repeatedly through recombination within the incompatibility loci in bifactorial (tetrapolar) Hymenomycetes (see Koltin, Stamberg \& Lemke, 1972, for review). Although a two-gene structure for the incompatibility locus of unifactorial fungi has never been detected, this possibility seems the most likely explanation for the non-parental types found in A. bitorquis. Proof of this possibility might be obtained by recovery of the two non-parental $A$ types from a single cross and regeneration of the two original parental types in a subsequent cross of the two non-parental types. A tetrad analysis might also indicate recombination, but such an analysis would be extremely difficult in this fungus.

The genetic control of spore viability remains to be determined. The wide range in incidence of spore germination (from $<5$ to $95 \%$ ) in different crosses under standard environmental conditions suggests a genetic basis that may be manipulated advantageously in breeding experiments. Analysis of appropriate crosses between progeny of parents with 
high and low potential for spore germination should clarify the number and nature of the genetic determinants for this character.

It is surprising that the first five collections of Agaricus from nature, obtained without any particular view to selection of members of the same species, are all interfertile. A continuing study lof wild Agaricus should reveal species distinct from A. bitorquis and perhaps one more closely related to A. bisporus.

These studies were supported at Harvard University by a gift from Campbell Soup Co. They were done in collaboration with my late husband Professor John R. Raper, deceased 2I May, 1974. The work was ably assisted by S. Carpenter, K. Kitazawa, J. Hendee, and E. Diala.

\section{REFERENCES}

BoIDIN, J. (I971). Nuclear behavior in the mycelium and the evolution of the Basidiomycetes. In Evolution in the Higher Basidiomycetes, pp. 129-146. Edited by R. H. Peterson. Knoxville: University of Tennessee Press.

Elliott, T. J. (1972). Sex and the single spore. Mushroom Science 8, I I-I 8.

EMERSON, S. (I949). Competitive reactions and antagonisms in the biosynthesis of amino acids by Neurospora. Cold Spring Harbor Symposia 14, 40-54.

Geisy, R. M. \& DAY, P. R. (I965). The septal pores of Coprinus lagopus (Fr.) sensu Buller in relation to nuclear migration. American Journal of Botany 52, 287-294.

Hughes, D. T. (1961). Chromosomes of the wild mushroom. Nature, London I90, 285-286.

Jı̌̆I, H. (I965). Cytological studies in the genus Agaricus. Mushroom Science 6, 80-83.

Koltin, Y., StamberG, J. \& Lemke, P. A. (I972). Genetic structure and evolution of the incompatibility factors in higher fungi. Bacteriological Reviews 36, I56-17I.

Miller, R. E. (I97I). Evidence of sexuality in the cultivated mushroom, Agaricus bisporus. Mycologia 63, 630-634.

Miller, R. E. \& Kananen, D. L. (1972). Bipolar sexuality in the mushroom. Mushroom Science 8, 7I 3-7I 8.

Monocha, M. S. (1965). Fine structure of the Agaricus carpophore. Canadian Journal of Botany 43, 1329I 333 .

Niederpruem, D. J. \& Wessels, J. G. H. (I969). Cytodifferentiation and morphogenesis in Schizophyllum commune. Bacteriological Reviews 33, 505-535.

Poppe, J. A. (1972). Un excellent Agaricus tetra-sporique cultivable commercialement avec succès. Mushroom Science 8, 517-525.

RAPER, C. A. (I975). The biology and breeding potential of Agaricus bitorquis. Mushroom Science 9 (in the Press).

RAPER, C. A. \& RAPER, J. R. (1966). Mutations modifying sexual morphogenesis in Schizophyllum. Genetics 54, I I 5 I-I I 68.

RAPER, C. A., RAPER, J. R. \& Miller, R. E. (I972). Genetic analysis of the life cycle of Agaricus bisporus. Mycologia 64, IO88-III7.

RAPER, J. R. (I963). Device for the isolation of spores. Journal of Bacteriology 86, 342-344.

RAPER, J. R. (1966). Genetics of Sexuality in Higher Fungi. New York: Ronald Press.

RAPER, J. R. \& RAPER, C. A. (1972). Life cycle and prospects for interstrain breeding in Agaricus bisporus. Mushroom Science 8, I-9. 